\title{
NOUVELLe
}

\section{Les décisions hâtives dans la schizophrénie sont fondées sur l'inférence circulaire}

Renaud Jardri ${ }^{1,2}$, Sophie Denève ${ }^{1}$
${ }^{1}$ Laboratoire de neurosciences cognitives, GNT (Group for neural theory), Inserm U960, École normale supérieure, 45, rue d'Ulm, 75005 Paris, France.

${ }^{2}$ SCALab (Sciences Cognitives et Sciences Affectives), Équipe PsyCHIC (Psychiatrie et Croyance), CNRS UMR9193, Université de Lille et Plateforme CURE (Centre Universitaire de Recherche et d'Exploration en psychiatrie), CHU de Lille, 59037 Lille, France.

renaud.jardri@chru-lille.fr
> Depuis plusieurs années, les grandes fonctions participant à nos interactions avec le monde (c'est-à-dire la perception, le contrôle moteur ou la prise de décision) sont considérées comme résultant d'un traitement probabiliste de l'information par le cerveau. Le cadre bayésien (inspiré du théorème de Bayes) a ainsi énormément influencé le champ des sciences cognitives, avec pour objectif de mieux comprendre le fonctionnement cérébral normal et pathologique. C'est ainsi qu'un trouble du traitement inférentiel de l'information a été proposé à l'origine de certains symptômes de la schizophrénie, tels que les hallucinations, le délire ou la désorganisation cognitive $[1,2]$. Le principal problème de ces théories bayésiennes repose sur la difficulté à pouvoir quantifier le lien existant entre les perturbations des mécanismes d'inférence et les symptômes psychotiques. C'est tout l'enjeu de la psychiatrie computationnelle, discipline naissante et ambitieuse, ayant pour objectif de mieux comprendre les maladies mentales par la modélisation des processus élémentaires de pensée et de leurs dysfonctionnements [3]. Cet article présente l'une de ces théories bayésiennes, intitulée «l'inférence circulaire», et son rationnel expérimental dans la schizophrénie.

L'idée de se référer à une approche bayésienne de la perception et de la décision dans la schizophrénie n'est pas nouvelle et a notamment été explorée à l'aide de tâches dites de raisonnement probabiliste (Figure 1). Devenue une référence dans le champ de la psychologie expérimentale, la «tâche des urnes», a notamment permis de montrer que les participants prenant des décisions hâtives, c'est-à-dire sur la base de moins d'information et avec une confiance accrue, présentaient davantage de symptômes délirants [4]. Notre équipe a récemment proposé une adaptation de cette procédure, intitulée la «tâche du pêcheur» (ou fisher task), permettant de quantifier l'importance accordée aux informations a priori, aux informations sensorielles entrantes et à leur combinaison optimale, dans la construction d'une croyance [5] ${ }^{l}$. À l'instar de sa grande sœur, la tâche du pêcheur capture bien le phénomène de conclusions hâtives, retrouvé plus prononcé chez les patients souffrant de schizophrénie, comparativement à des témoins sains, appariés en âge et en niveau d'éducation. À l'aide d'un modèle informatique appliqué aux données issues de la tâche du pêcheur, nous avons récemment tenté d'identifier les mécanismes inférentiels à l'origine des comportements observés. « L'inférence circulaire » est un modèle bayésien hiérarchique: il s'appuie sur l'hypothèse que le cerveau est un système hiérarchique [6], dont les unités élémentaires (comme les neurones, les aires corticales, etc.) tentent de prédire leurs entrées, en intégrant les informations nouvelles à celles déjà connues (nos connaissances a priori sur le monde). On peut également parler

\footnotetext{
${ }^{1}$ Les participants étaient invités à évaluer la probabilité qu'un poisson rouge tout juste pêché, provienne d'un des deux lacs présentés à l'écran (le gauche ou le droit). Chaque essai commençait par la présentation de deux paniers de tailles différentes, chacun associé à un lac. Les participants devaient interpréter la taille relative des paniers comme représentant la préférence du pêcheur pour chaque lac.
}

de modèle d'inférence causale [7] pour caractériser cette famille de modèles. Après échange d'informations, le réseau converge vers une représentation ou une croyance globale cohérente. Un des éléments cruciaux au bon fonctionnement de ces modèles, repose sur le contrôle précis du flux des messages envoyés dans la hiérarchie corticale. En effet, la forte connectivité intrinsèque existant entre les différents éléments du réseau, expose à un risque de redondance d'une même information.

Les informations sensorielles sont propagées dans la hiérarchie corticale, depuis les aires sensorielles primaires (le lobe occipital dans le cas de la vision) vers des aires qui interprètent et exploitent ces informations (les aires frontales, temporales, motrices, etc.) afin de contrôler le comportement [8] $(\rightarrow)$. Le problème est

$(\rightarrow)$ Voir la Nouvelle de $\varepsilon$. Fino, $m / s n^{\circ} 3$, mars 2012, page 242

que ces aires corticales de haut-niveau envoient également de nombreuses connexions descendantes vers les aires occipitales, exerçant ainsi une forte influence sur les réponses sensorielles. De ces flux ascendants et descendants, peut résulter une «circularité » de l'information, avec des prédictions a priori influençant les représentations sensorielles qui, à leur tour, changent les prédictions, etc. En l'absence de mécanisme de contrôle adéquat, cette circularité force le système à construire des croyances, certes fortes, mais pour autant fausses (car excessives). Un tel processus pourrait, par exemple, mener le système à interpréter du bruit sensoriel comme une preuve de la présence 


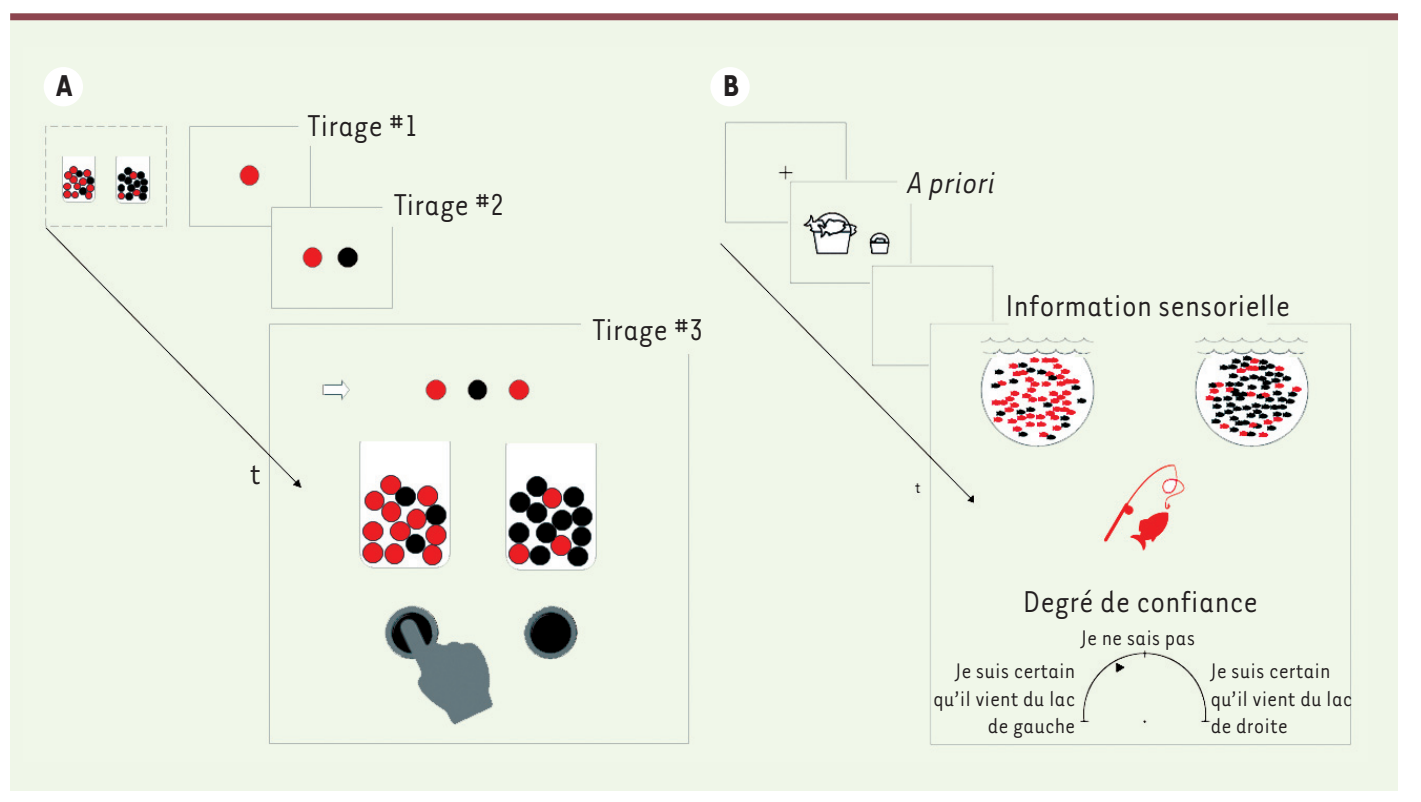

Figure 1. Tâches de raisonnement probabiliste utilisées pour explorer les décisions hâtives. A. La tâche des urnes. Deux urnes contenant un ratio inverse de boules rouges et noires sont présentées au participant. À chaque essai, une boule est tirée en aveugle de l'une des deux urnes (toujours la même). Lorsque le participant pense savoir de quelle urne proviennent les boules, il répond en appuyant sur le boitier réponse indiquant la droite ou la gauche. Les sujets délirants ont tendance à prendre des décisions hâtives (jumping-to-conclusion), c'est-à-dire fondées sur moins de preuves et avec un niveau de confiance plus important. $\boldsymbol{B}$. La tâche du pêcheur. Il s'agit d'une variante de la tâche des urnes, décrite par Jardri et al. en 2017 [5]. Ici, il n'y a qu'un seul tirage mais une information a priori est fournie aux participants. Le sujet doit rapporter son degré de confiance dans le fait que le poisson vienne de l'un des deux lacs. Chaque essai débute par la présentation d'une information a priori (à savoir, deux paniers de pêcheur), représentant la chance que le poisson, ensuite présenté, vienne du lac de droite ou du lac de gauche. Après retrait de cette information, les lacs sont présentés, avec un ratio inverse de poissons noirs/rouges. La réponse du participant est recueillie sur une échelle semi-circulaire indiquant son niveau de confiance (allant de «je ne sais pas » à « je suis sûr qu'il s'agit du lac de droite/ de gauche »).

d'un objet, ou encore à percevoir un objet simplement imaginé.

Afin d'éviter qu'une même information ne soit donc comptabilisée plusieurs fois, des boucles inhibitrices, présentes à chaque étage de la hiérarchie, sont nécessaires pour supprimer ces informations redondantes, qu'elles soient ascendantes (c'est-à-dire allant des entrées sensorielles aux aires de plus haut-niveau), ou descendantes (les projections du haut vers le bas). II existe dans le cerveau, une signature très forte de ces boucles inhibitrices sous forme d'une balance entre l'excitation et l'inhibition. En effet, à chaque activation, tout neurone voit augmenter non seulement son excitation (sous forme de courant dépolarisant), mais également son inhibition (courant hyperpolarisant). Les deux s'annulent presque, impliquant le fait que les inter-neurones inhibiteurs sont capables de supprimer l'information redondante (et donc pré- visible) dans les entrées ascendantes et descendantes. En altérant la balance entre l'excitation et l'inhibition, donc le contrôle de ces boucles, une même information pourrait être comptée plusieurs fois, menant à des croyances exagérément fortes, ainsi qu'à une augmentation artificielle du poids donné aux informations nouvelles par rapport aux croyances internes, ou vice-versa. On parle alors d' « inférence circulaire» $[2,9]$. Des simulations mathématiques de ce phénomène d'inférence circulaire montrent qu'il peut induire des aberrations perceptives (comme des hallucinations), des fausses croyances (telles des délires, des interprétations et des décisions hâtives), ou amener à la construction de croyances opposées mais de force égale (comme la pensée désorganisée).

En appliquant le modèle de l'«inférence circulaire » aux données de la tâche du pêcheur, nous avons pu mon- trer que la prise de décision hâtive, observée chez les patients souffrant de schizophrénie, était liée à une amplification importante des informations sensorielles entrantes, via une altération des boucles inhibitrices contrôlant le flux ascendant [5]. Le degré d'altération des boucles inhibitrices varie d'un sujet à l'autre, mais il reste fortement corrélé à la rigidité des croyances, que celles-ci soient pathologiques (délire) ou subcliniques (Figure 2). De manière intéressante, cette amplification de l'information sensorielle, pouvant être à l'origine des symptômes psychotiques, est compatible avec l'observation d'une diminution de la susceptibilité aux illusions perceptives, chez les patients avec des symptômes positifs de schizophrénie [10]. En effet, la théorie bayésienne de la perception attribue les illusions à l'effet d'un a priori suffisamment fort pour biaiser les interprétations senso- 
A

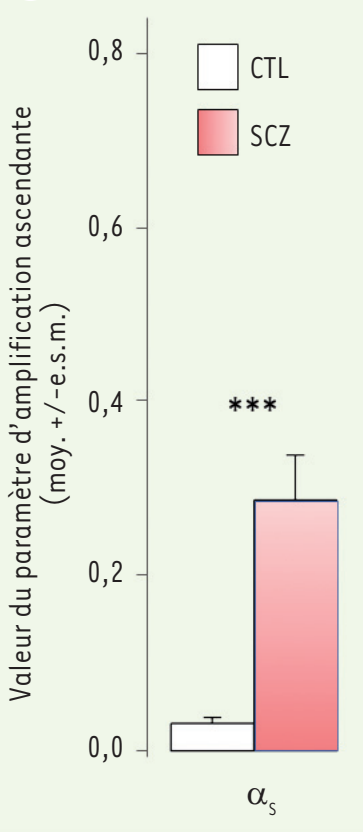

B

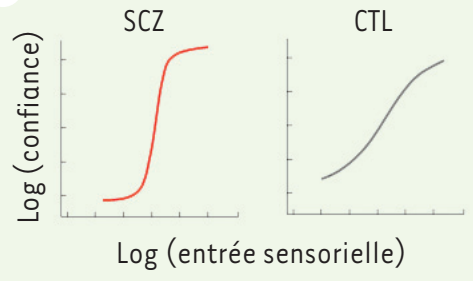

c

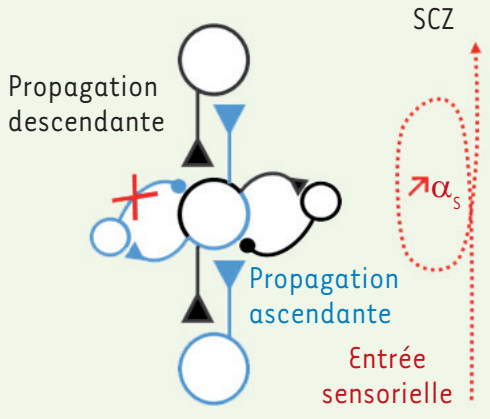

D

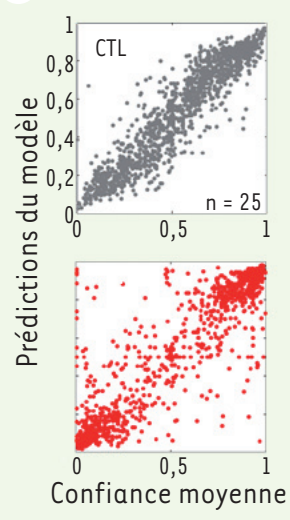

$\varepsilon$

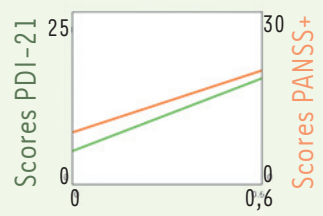

Figure 2. Le modèle de l'inférence circulaire capture de manière optimale le niveau de confiance des participants soumis à la tâche du pêcheur. A. Le comportement des patients est lié à une amplification aberrante de l'information sensorielle entrante $\left(\alpha_{s}\right)$. B. Comparativement aux témoins sains (CTL, en noir), les patients souffrant de schizophrénie se fondent davantage sur l'information sensorielle, en suivant une règle d'amplification sigmoïdale ( $S C Z$, en rouge), telle

que prédite par le modèle d'inférence circulaire $(C, D)$ Le modèle de l'inférence circulaire capture ce phénomène au sein des deux populations de participants (CTL et SCZ), et ce malgré leur grande hétérogénéité. $\varepsilon$. Cette amplification $\alpha$ est associée à la force des croyances aberrantes, cliniques (via les scores de PANSS positive dans la population SCZ, en orange) et subcliniques (via l'utilisation de l'échelle PDI-2l chez l'ensemble des participants, en vert). PANSS : positive and negative syndrome scale ; PDI-21 : Peters et al. delusions inventory, un questionnaire conçu pour mesurer l'intensité des croyances normales et pathologiques.

rielles. Une amplification incontrôlée des informations ascendantes (et donc une confiance accrue dans les informations sensorielles «brutes») affaiblirait l'illusion. L'altération des boucles inhibitrices ascendantes, observées dans la schizophrénie, «immuniserait » en quelque sorte les patients contre ce type de fausse perception, tout en les exposant au risque d'hallucinations et de croyances délirantes. Globalement, I'inférence circulaire est capable de capturer la grande hétérogénéité clinique et comportementale observée dans les tâches de raisonnement probabiliste. Par exemple, une altération sélective des boucles inhibitrices contrôlant le flux ascendant est corrélée à la sévérité des symptômes psychotiques. Cependant l'implémentation neurale précise des boucles inhibitrices restent à déterminer [11]. $\diamond$ Jumping-to-conclusions in schizophrenia is mediated by circular inference

\section{LIENS D'INTÉRÊT}

Les auteurs déclarent n'avoir aucun lien d'intérêt concernant les données publiées dans cet article.

\section{RÉFÉRENCES}

1. Fletcher PC, Frith CD. Perceiving is believing: a Bayesian approach to explaining the positive symptoms of schizophrenia. Nat Rev Neurosci 2009 ; $10: 48-58$.

2. Jardri $R$, Deneve $S$. Circular inferences in schizophrenia. Brain 2013; $136: 3227-41$.

3. Stephan KE, Mathys C. Computational approaches to psychiatry. Curr Opin Neurobiol 2014 ; 25 : 85-92.
4. Moritz S, Woodward TS. Jumping to conclusions in delusional and non-delusional schizophrenic patients. BrJ Clin Psychol $2005 ; 44$ : 193-207.

5. Jardri R, Duverne $S$, Litvinova AS, et al. Experimental evidence for circular inference in schizophrenia. Nat Commun $2017 ; 8: 14218$.

6. Friston K. Hierarchical models in the brain. PLoS Comput Biol 2008 ; 4 : el000211.

7. Lochmann T, Deneve S. Neural processing as causal inference. Curr Opin Neurobiol 2011 ; 21 : 774-81.

8. Fino $\varepsilon$. Les connexions neuronales en pleine lumière L'organisation des réseaux inhibiteurs dans le néocortex. Med Sci (Paris) 2012 ; 28 : 242-5.

9. Denève $S$, Jardri R. Circular inference: mistaken belief, misplaced trust. Curr Opin Behav Sci 2016;11:40-8.

10. Notredame CE, Pins D, Devene $S$, et al. What visual illusions teach us about schizophrenia. Front Integr Neurosci $2014 ; 8: 63$.

11. Jardri R, Hugdahl K, Hughes M, et al. Are hallucinations due to an imbalance between excitatory and inhibitory influences on the brain? Schizophr Bull 2016; 42 : 1124-34.

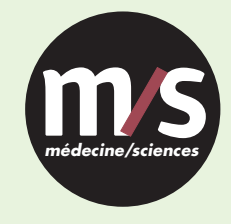

Tarifs d'abonnement $\mathrm{m} / \mathrm{s}-2017$

Abonnez-vous

à médecine/sciences
$>$ Grâce à $m / s$, vivez en direct les progrès des sciences biologiques et médicales

Bulletin d'abonnement page 1010 dans ce numéro de $\mathrm{m} / \mathrm{s}$

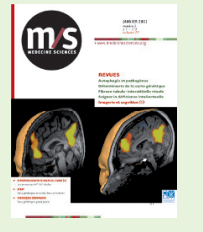

